\title{
Classification and localisation of carcinoembryonic antigen (CEA) related antigen expression in normal oesophageal squamous mucosa and squamous carcinoma
}

\author{
D S A Sanders, C A Wilson, F J Bryant, J Hopkins, G D Johnson,.D M Milne, M A Kerr
}

Department of Pathology, The Medical School, University of Birmingham, Birmingham D S A Sanders C A Wilson F J Bryant

Department of Medicine, Queen Elizabeth Hospital, Birmingham

J Hopkins

Department of Immunology, The Medical School, University of Birmingham, Birmingham G D Johnson

Department of Pathology, Ninewells Hospital and Medical School, Dundee D M Milne

M A Kerr

Correspondence to: Dr D S A Sanders Department of Histopathology, Selly Oak Hospital, Raddlebarn Road Birmingham M29 6JD.

Accepted for publication 29 November 1993

\begin{abstract}
Using a panel of carcinoembryonic antigen (CEA) related antibodies in normal oesophageal squamous mucosa CEA expression is present on suprabasal squames localised to the cell membrane. Immunoblotting shows that this positivity is predominantly due to a glycoprotein of around $180 \mathrm{kDa}$ representing CEA itself. Positivity in squamous carcinomas is confined to cells in foci of squamous differentiation. A shift from membranous localisation to predominant cytoplasmic overexpression is shown between normal and malignant squames using confocal microscopy. The recognition of an adhesive role for CEA and a role in enhancing distant metastases in those tumours expressing CEA highlights the importance of recording CEA expression and changes in subcellular distribution between normal and malignant tissues; CEA expression in oesophageal squamous mucosa has not been well recognised previously and changes in expression may prove of great significance in the spread and dissemination of squamous carcinoma.

(Gut 1994; 35: 1022-1025)
\end{abstract}

Recent advances in molecular biology and gene cloning have resulted in great improvements in the understanding of the carcinoembryonic antigen (CEA) related family of glycoproteins leading to a reassessment of the clinical outlook for CEA and giving insight into the function of these molecules. ${ }^{1}$ The CEA gene family is now known to belong to the immunoglobulin superfamily and in particular the C-2 set of adhesion molecules; cell adhesion properties are well established for CEA and its classic related cross reacting antigens, non-specific cross reacting antigen (NCA), and biliary glycoprotein. Binding may be homophilic (CEA to CEA) or heterophilic (NCA to CEA), is neither calcium or temperature dependent, and is capacitated by membranous expression on cells. ${ }^{2}$

In the gastrointestinal tract most attention has focused on CEA expression in the normal colonic and gastric mucosa and adenocarcinomas. CEA expression in squamous mucosa and squamous carcinoma is not widely recognised and has received little attention. We recently reported expression of a CEA related antigen on the cell surface of squames in normal cervical mucosa, which suggests a key role in maintaining the integrity of the mucosa through an adhesive function. ${ }^{3}$ Any change in the pattern of expression and subcellular distribution of CEA, as has been reported between normal colonic mucosa and adenocarcinoma, ${ }^{45}$ may be of significance in tumour dissemination.

We therefore have now extended our previous study to classify and localise CEA related antigen positivity in normal

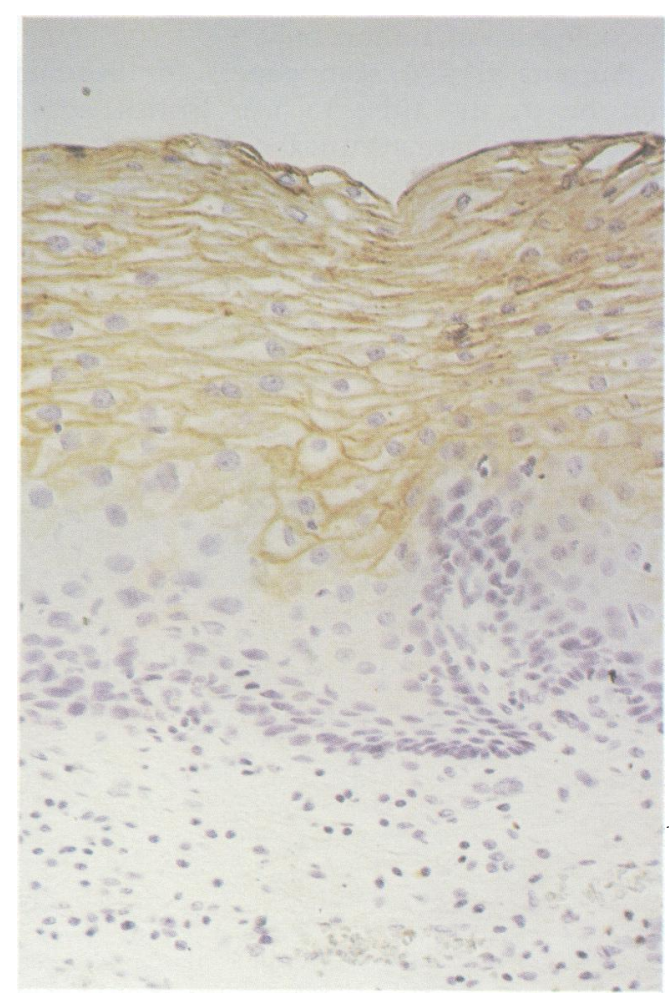

Figure 1: Normal oesophageal squamous mucosa stained with polyclonal anti-CEA. Note the membranous positivity on suprabasal squames with an unstained basal zone (original magnification $\times 40$ ). 


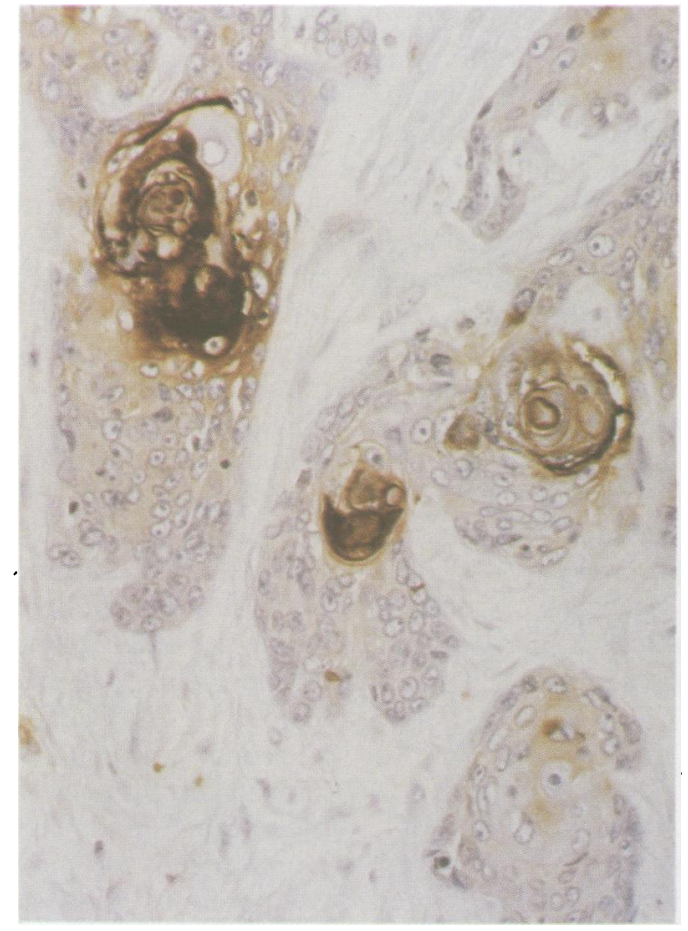

Figure 2: Oesophageal squamous carcinoma stained with polyclonal anti-CEA. Note strong cytoplasmic staining of cells at the centre of epithelial pearls (original magnification $\times 250$ ).

oesophageal squamous mucosa and squamous carcinoma.

\section{Methods}

IMMUNOHISTOCHEMISTRY

Sections cut from archive paraffin wax blocks from 30 cases of invasive oesophageal squamous carcinoma and from 20 blocks of endoscopic biopsy specimens of normal oesophageal mucosa from non-cancer patients were pretreated with $0.1 \%$ trypsin in

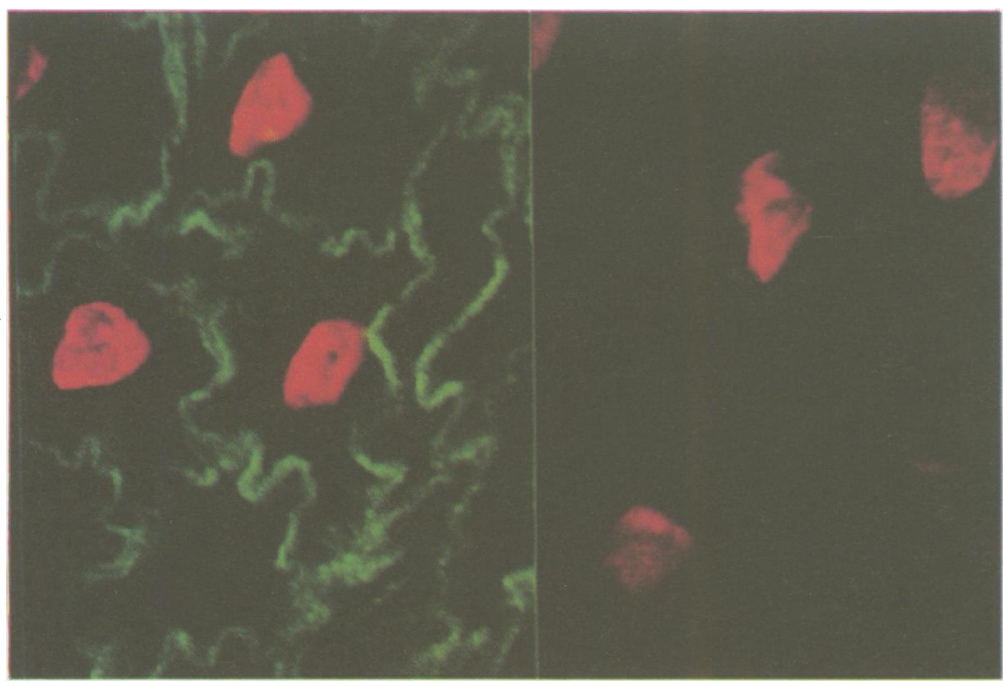

Figure 3: Confocal microscopy of normal oesophageal mucosa. A merged image of the red channel (nuclear counterstain) and the green channel (CEA related fluorescence) shown in an optical section through the centre of the nucleus. In the test section (left field) CEA related fluorescence (green) is clearly localised to the cell membrane with no cytoplasmic positivity shown. No background fluorescence is seen in control sections (right field) (original magnification $\times 400$ ).

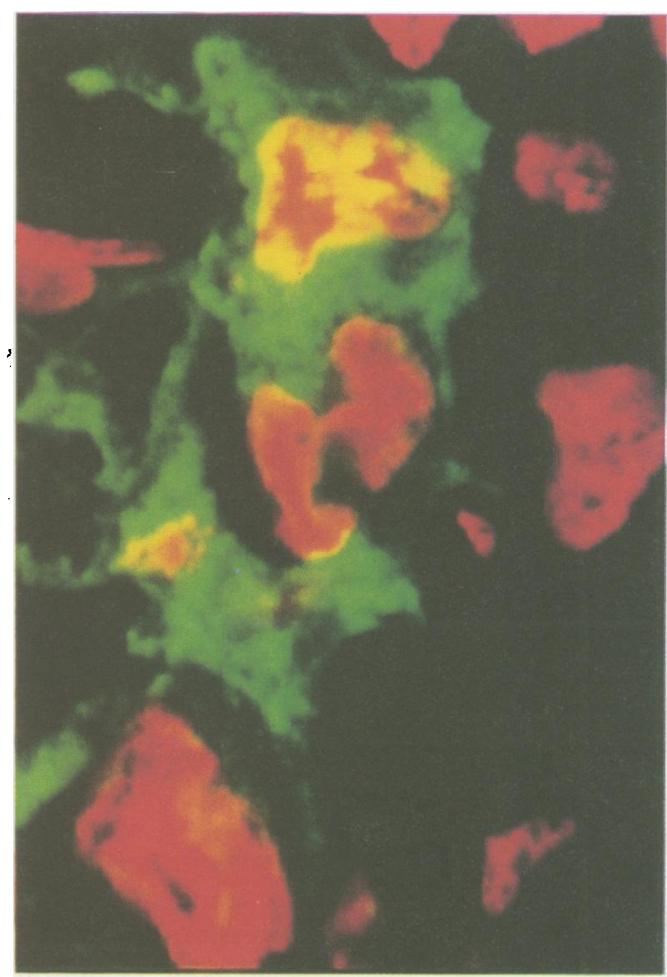

Figure 4: Confocal microscopy of a group of invasive cells in an oesophageal squamous carcinoma. A merged image of the red channel (nuclear counterstain) and the green channel (CEA related fluorescence) in an optical section through the centre of the nucleus. CEA related fluorescence is seen in the cell cytoplasm (green) and admixed with degenerate nuclear material (gold). No membrane associated positivity is seen in comparison with Figure 3 (original magnification $\times 400$ ).

TRIS buffered saline at $\mathrm{pH} 7.6$ at room temperature for 25 minutes and stained with a panel of anti-CEA antibodies - polyclonal anti-CEA (DAKO) at 1/200 dilution; monoclonal anti-CEA A5B7 (DAKO) at 1/50 dilution; monoclonal anti-CEA IM10 $6 \cdot 2$ (Scottish Antibody Production Unit) 1/50 dilution.

All sections were developed with the appropriate second antibody and an ABC Vectastain kit (Vector Laboratories, Peterborough). Control sections were prepared by missing out the anti-CEA.

IMMUNOBLOTTING

Fresh normal mid-oesophageal endoscopic biopsy specimens were used from two patients. Tissue was finely chopped resuspended in phosphate buffered saline at a concentration of $100 \mathrm{mg}$ wet tissue/ml and then homogenised using a Polytron homogeniser at half speed for two minutes. Triton X-100 was added to a final concentration of $1 \% \mathrm{vol} / \mathrm{vol}$ and the extract rotated for one hour at $4^{\circ} \mathrm{C}$. After centrifugation the supernatent was removed for further study. Proteins were separated by SDS-polyacrylamide gel electrophoresis on $10 \%$ polyacrylamide gels by the method of Laemmli ${ }^{6}$ and stained with polyclonal anti-CEA. Extracts of neutrophil membrane were run in adjacent lanes as a positive control (prepared as in reference 7 ). 


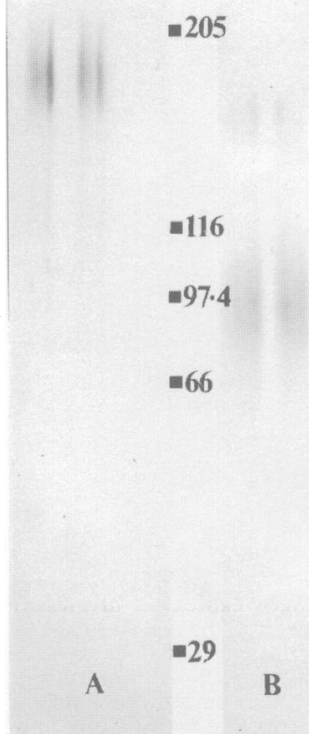

Figure 5: Immunoblot of two samples of oesophageal squamous mucosa extract (A) and neutrophil membrane extract (B) stained with polyclonal stained with polyclonal broad band at around 180 $k D a$ for oesophagus with bands at around 100 and $160 \mathrm{kDa}$ for neutrophils.

\section{CONFOCAL MICROSCOPY}

Six $\mu \mathrm{m}$ sections were cut from paraffin wax blocks of four cases of normal squamous mucosa and four squamous carcinomas. Sections were incubated in methanol in $0.3 \%$ hydrogen peroxide at room temperature, washed, and trypsinised. Sections were incubated with $10 \%$ normal goat serum, washed, and incubated with polyclonal antiCEA at 1 in 200 dilution. After further washing the fluorescent conjugate was added 1:40 in phosphate buffered saline. Nuclei were counterstained with propidium iodide and sections mounted in Glycergel (DAKO). Control sections were prepared by missing out the anti-CEA. Sections were viewed on a Bio-Rad 500 Laser Scanning Confocal Microscope System attached to a Leitz SM-Lux fluorescence microscope.

\section{Results}

\section{IMMUNOHISTOCHEMISTRY}

In normal squamous mucosa CEA related expression was seen on the cell membrane of a broad zone of suprabasal squames with both the polyclonal and monoclonal anti-CEAs. A zone of basal cells was unstained (Fig 1).

In squamous carcinoma CEA related positivity was seen in cells showing the features of squamous cells at the centre of epithelial 'pearls' (Fig 2). Little expression was seen in poorly differentiated carcinomas except in occasional single cells.

\section{CONFOCAL MICROSCOPY}

CEA related fluorescence was clearly shown localised to the cell membrane in normal oesophageal mucosa; no fluorescence was seen in control sections. No cytoplasmic expression was seen (Fig 3). In contrast strong predominantly cytoplasmic CEA related fluorescence was seen in CEA expressing cells in squamous carcinoma (Fig 4). Focal membranous staining was also seen.

\section{IMMUNOBLOTTING}

A strong broad CEA related band was seen at around $180 \mathrm{kDa}$ in normal oesophageal squamous mucosa known to represent CEA itself. In contrast bands were seen at around $100 \mathrm{kDa}$ and $160 \mathrm{kDa}$ in neutrophil membrane extract known to represent NCAs ${ }^{7}$ (Fig 5).

\section{Discussion}

The use of an unabsorbed polyclonal anti-CEA that recognises CEA and NCA in conjunction with monoclonal antibodies, which should be more specific for CEA gives valuable information about CEA related immunoreactivity in paraffin processed tissues. Immunoblotting permits more precise classification of CEA related expression in a tissue by identifying the molecular weight of the predominant glycoproteins expressed.

We have shown that the predominant CEA related antigen in oesophageal mucosa is a glycoprotein of around $180 \mathrm{kDa}$ known to represent a true CEA. A broad band is seen on immunoblotting because the polyclonal antiCEA recognises CEAs with subtle differences of glycosylation and hence molecular weight; the core peptide of CEA itself is only $80 \mathrm{kDa}$, carbohydrate structures accounting for up to $60 \%$ of the mass of the mature glycoprotein. Variable glycosylation is also responsible for much of the complex immunogenicity of the CEA superfamily of molecules. CEA of 180 $\mathrm{kDa}$ is also expressed in normal cervical squamous mucosa but is slightly higher than that expressed by standard adenocarcinoma $(165 \mathrm{kDa}) .^{3}$ Immunoblotting of adenocarcinoma shows additional bands from $55-120 \mathrm{kDa}$ and in cervical squamous mucosa $18 \cdot 5-66 \mathrm{kDa}$ glycoproteins have been reported representing various NCAs. ${ }^{38}$ CEA 180 acts as an accessory adhesion molecule mediating interactions between tumour cells and endothelial basement membrane $^{9}$ and CEA interacts with liver Kupffer cells promoting distant metastasis. ${ }^{10}$ Membranous expression of CEA in the normal oesophagus suggests a key adhesive part in maintaining the integrity of the mucosa. This is in contrast with CEA expression in the normal colon, which is on the luminal surface of cells and therefore has little part to play in maintaining mucosal integrity. ${ }^{4}$ Bacterial colonisation in the gut is known to be influenced, however, by bacterial ligand-CEA interactions. ${ }^{11}$

Expression of CEA on suprabasal (mature) squames and restricted to cells in squamous pearls in oesophageal carcinoma confirms that, as in the cervix, CEA expression, in particular membranous expression, is a marker of squamous differentiation. ${ }^{12}$ Using confocal microscopy we have shown that there is a change from membranous to predominantly cytoplasmic overexpression between normal and neoplastic squames. The differences in tissue specific CEA expression can largely be explained by the finding of widely varying levels of transcription activity within the promoter region of the CEA genes, although control of this element is poorly understood. ${ }^{13}$ Anchorage of CEA into the cell membrane is through replacement of the hydrophobic $M$ domain on CEA with a glycosyl phosphatidylinositol moiety. Membrane bound CEA can be released through the action of specific phospholipases and it is possible that these two mechanisms interact to determine the amount of CEA expressed on cells or released into the serum, or both. ${ }^{14}$ Raised serum concentrations of CEA have been noted in squamous carcinomas. ${ }^{15} 16$

Membranous upregulation of CEA is recognised in colonic adenocarcinoma and hence CEA may have a role in promoting the metastasis of adenocarcinoma. In squamous carcinoma loss of CEA expression and a shift to cytoplasmic expression may adversely influence cellular cohesion; CEA could only have a putative influence in the metastasis of squamous carcinoma in tumours where a significant proportion of cells show membranous expression. The absence of CEA 
from cells at the periphery of differentiated squamous tumour islands means that CEA could have little influence on interactions at the tumour/stroma interface.

Increasingly tumours other than adenocarcinomas are being recognised as expressing CEA related antigens. ${ }^{12}$ The development of monospecific anti-CEA antibodies reacting with CEA on the surface of tumour cells has led to the successful immunolocalisation of CEAexpressing tumours and their metastases. ${ }^{17}$ Early work has suggested a role for CEA antibodies in tumour immunotherapy. ${ }^{18}$ This highlights the importance of localising and typing CEA related antigens in normal and neoplastic squamous tissues.

We wish to thank Mr Barrie Sims for the photomicrograph of the immunoblot. This work was funded by the United Birmingham Hospitals Endowment Fund (F08689).

1 Thompson JA, Grunert F, Zimmermann W. Carcinoembryonic gene family: molecular biology and clinical perspectives. F Clin Lab Anal 1991; 5: 344-66.

2 Oikawa S, Inuzuka C, Kuroki M, Matsuoka Y, Kosaki G, Nakazato $H$. Cell adhesion activity of non-specific crossreacting antigen (NCA) and carcinoembryonic antigen (CEA) expressed on CHO cell surface: homophilic and heterophilic adhesion. Biochem Biophys Res Commun 1989; 164: 39-45.

3 Sanders DSA, Stocks SC, Milne DM, Milne GA, Hopwood D, Kerr MA. Membranous expression of carcinoembryonic antigen (CEA) in the normal cervical squamous onic antigen (CEA) in the norm

mucosa. F Pathol 1991; 167: 77-82.
4 Ahnen DJ, Nakane PK, Brown WR. Ultrastructural localisation of carcinoembryonic antigen in the normal intestine and colon cancer. Cancer 1982; 49: 2077-90.

5 Wolf B, Thompson JA, von Kleist S. Differences in the ultrastructural localisation of carcinoembryonic antigen in human breast and colon tumour cells. Tumour Biol 1984; 5: 233-42.

6 Laemmli UK. Cleavage of structural proteins during assembly of bacteriophage T4. Nature 1970; 227: 680-5.

7 Stocks SC, Albrechtsen M, Kerr MA. Expression of the CD15 differentiation antigen (3-fucosyl-N-acetyl-lactosamine, Lex) on putative neutrophil adhesion molecules tosamine, Lex) on putative neutrophil adhesion molect

8 Chana T, Han X, Dagg B, et al. TDM 35 - A new monoclonal antibody to the XH1 cervical carcinoma cell line. Characterisation and immunoperoxidase localisation in benign and malignant tissues. $\mathscr{F}$ Pathol 1992; 167: 391-7.

9 Pignatelli M, Durbin H, Bodmer WF. Carcinoembryonic antigen functions as an accessory adhesion molecule mediating colon epithelial cell-collagen interactions. Proc Natl Acad Sci USA 1990; 87: 1541-5.

10 Hostetter RB, Cambell DE, Chi K. Carcinoembryonic antigen enhances the metastatic potential of human colorectal carcinoma. Arch Surg 1990; 125: 300-4.

11 Leusch HG, Drzeniek Z, Hefta SA, Markos-Putztai Z, Wegener $C$. The putative role of members of the CEAgene family (CEA, NCA and BGP) as ligands for the bacterial colonisation of different human epithelial tissues. Int $\mathcal{F}$ Med Microbiol 1991; 275: 118-22.

12 Sanders DSA, Ferryman SR, Bryant FJ, Rollason TP. Patterns of CEA-related antigen expression in invasive squamous carcinoma of the cervix. $\mathcal{F}$ Pathol 1993; 171: 21-6.

13 Schrewe H, Thompson J, Bona M, Hefta LJF, Maruya A, Hassauer $M$, et al. Cloning of the complete gene for the carcinoembryonic antigen: analysis of its promoter indicates a region conveying cell type-specific expression. $\mathrm{Mol}$ Cell Biol 1990; 10: 2738-48.

14 von Kleist S, Wittekind C, Sandritter W, Gropp H. CEA positivity in sera and breast tumor tissues obtained from

15 van Nagell JR, Meeker WR, Parker JC, Harralson JD. Carcinoembryonic antigen in patients with gynaecologic malignancy. Cancer 1875; 35: 1372-6.

16 Kjorstad KE, Orjasaester H. The prognostic value of CEA determinations in the plasma of patients with squamous cell cancer of the cervix. Cancer 1982; 50: 283-7.

17 Hammarstrom S, Shively JE, Paxton RJ, Beatty BG, Larsson A, Ghosh R, et al. Antigenic sites in carcinoembryonic antigen. Cancer Res 1989; 49: 4852-8.

18 Goldenburg DM. Current status of cancer imaging with radiolabelled antibodies. $\mathcal{f}$ Cancer Res Clin Oncol 1987; 113: 203-8. 